\section{Homöopathie: Beobachtungsstudien bei niedergelassenen Praktikern: Ein erster Erfolg mit IIPCOS-1}

\section{Riley D, Fischer M, Singh B, Haidvogl M, Heger M: Homeopathy and conventional Medicine: An outcomes study comparing effectiveness in a primary care setting. J Altern Complement Med 2001;7:149-159}

Background: Recent meta-analyses of randomized controlled trials in homeopathy have suggested that homeopathy is more than a placebo response.

Objective: Comparison of the effectiveness of homeopathy in primary care with conventional medicine in primary care for three commonly encountered clinical conditions.

Design: An international mulitcenter, prospective, observational study in real world medical setting comparing the effectiveness of homeopathy with conventional medicine.

Participants: 30 investigators with conventional medical licenses at six clinical sites in four countries enrolled 500 consecutive patients with at least one of the following three complaints: i) upper respiratory tract complaints including allergies; ii) lower respiratory tract complaints, including allergies; or iii) ear complaints.

Main Outcome Measures: The primary outcomes criterion was the response to treatment, defined as cured or major improvement after 14 days of treatment. Secondary outcomes criteria were: i) rate of recovery; ii) occurrence of adverse events; iii) patient satisfaction; and iv) length of consultation.

Results: 456 patient visits were compared: 281 received homeopathy, 175 received conventional medicine. The response to treatment as measured by the primary outcomes criterion for patients receiving homeopathy was $82.6 \%$, for conventional medicine it was $68 \%$. Improvement in less than 1 day and in 1-3 days was noted in $67.3 \%$ of the group receiving homeopathy and in $56.6 \%$ of those receiving conventional medicine. The adverse events for those treated with conventional medicine was $22.3 \%$ versus $7.8 \%$ for those treated with homeopathy. $79.0 \%$ of patients treated with homeopathy were very satisfied, and $65.1 \%$ of patients treated with conventional medicine were very satisfied. In both treatment groups $60 \%$ of cases had consultations lasting between 5 and $15 \mathrm{~min}$.

Conclusions: Homeopathy appeared to be at least as effective as conventional medical care in the treatment of patients with the three conditions studied.

\section{Kommentar - C. Güthlin, Freiburg i.Br.}

Die Studie von David Riley et al. ist ein ermutigendes Beispiel im Bereich der anwendungsorientierten Beobachtungs- studien in der niedergelassenen Praxis. Ermutigend nicht nur wegen der Ergebnisse (adjustierte Odds-Ratio von 1,96 zu Gunsten der Homöopathie), Mut macht vor allem, dass es der IIPCOS-1 (International Integrative Primary Care Outcomes Study) erstmalig gelungen ist, relativ viele Patientenkontakte aus der homöopathischen Praxis $(\mathrm{n}=281)$ mit Kontakten aus der konventionellen Praxis $(n=175) z u$ vergleichen. Dabei wurden zentrale Bedingungen einer angemessen «Outcomes»Forschung umgesetzt: Es handelt sich um ein prospektives Design, das ohne grosse Einschränkungen bezüglich der Medikation und der Patientenselektion auskommt, die in der homöopathischen Praxis relevanten Beschwerden der oberen und unteren Atemwege sowie Ohrenschmerzen prüft und die Verbesserung oder auch Gesundung der Patienten aus deren Sicht (gemessen mit der Glasgow Homeopathic Hospital Outcome Scale) als Hauptzielkriterium misst. Die Katamnesen sind mit 14-tägig und 28-tägig anberaumter Telefonbefragung aussergewöhnlich sorgsam geplant. Darüber hinaus konnten international Praxen für diese Multicenter-Studie gewonnen werden. $\mathrm{Zu}$ erwähnen bleibt eine Einschränkung bezüglich des Designs: Die Auswertung erfolgte nicht «intent-to-treat», da 41 Patienten ausgeschlossen wurden, für die keine Followup-Befragung vorlag.

Weitere einzelne Aspekte sollen ebenfalls etwas genauer unter der Lupe genommen werden. Einmal ist zu sagen, dass gerade die Atemwegserkrankungen in der konventionellen Praxis meist mit antibiotischer Therapie bekämpft werden, eine Therapie, die etwa im Falle von Viruserkrankungen wirkungslos bleiben muss und nicht ohne Nebenwirkungen ist. Tatsächlich zeigte sich in dieser Studie eine deutlich höhere Rate an Nebenwirkungen unter den konventionell Behandelten aufgrund des Antibiotikaeinsatzes bei gleichzeitigem Einfluss auf die Ergebnisse. Patienten, die keine Nebenwirkungen erfuhren, sprachen besser auf die Therapie an und waren mit ihr zufriedener.

Insgesamt sind vielleicht die Atemwegserkrankungen (akut und atopisch) so etwas wie «Paradebeispiele», da die Homöopathie einerseits aus eigener Sicht diese zu therapieren vermag und andererseits keine hoch wirksamen adäquaten Medikamente auf Seiten der Standardtherapie zur Verfügung stehen. Für das Selbstbild der Homöopathen spricht an dieser Stelle, dass in der Tat in der vorliegenden Studie deutlich mehr Patienten innerhalb des ersten Tages auf die homöopathische Therapie mit Verbesserung reagierten als dies bei konventioneller Behandlung der Fall war.

Aus homöopathischer Sicht fällt weiter auf, dass zwar keine Vorgaben bezüglich des zu verwendenden homöopathischen Präparats gemacht wurden, jedoch nur Potenzen von C30 zugelassen waren. Damit ist implizit nur akute Therapie möglich, obwohl explizit auch der Einschluss von allergischen Erkrankungen genannt ist. Leider wird dem Leser nicht transparent, wie es zu dieser Vorgabe kam.

Eine Analyse der sonstigen Medikation ergab, dass 16\% der homöopathischen Patienten auch konventionelle Medikamen- 
te bekamen, aber nur 4,6\% der konventionell Behandelten zusätzlich homöopathische Präparate. Damit erhalten wir ein realistisches Abbild der homöopathischen Praxis, das nichts an der oben erwähnten höheren Wirksamkeit bezüglich der Beschwerden ändert. An dieser Stelle wird jedoch deutlich, dass wir hier von einer Evaluation von Homöopathen in der Praxis sprechen, nicht von der Homöopathie per se. Oder um es mit den Autoren zu sagen: Es ging um die Frage, ob die Homöopathie eine hilfreiche Form der Behandlung innerhalb des Gesundheitssystems ist, und nicht um die Frage, ob sich Homöopathie letzten Endes als Placebo erweist.

Methodisch wurde den deutlichen Unterschieden in den beiden betrachteten Gruppen mit adjustierten Odd-Ratios und Subgruppenanalysen Rechnung getragen, wobei letztere zu teilweise sehr kleinen (und damit bedeutungslosen) Gruppengrössen führte. Nicht nur deshalb sei an dieser Stelle der Wunsch «Weiter so!» geäussert. Gerade diese Art von Studien erfordert grosse Gruppen, vor allem sollte der Versuch gemacht werden, gleich grosse Gruppen zu erzeugen. Da gerade die Bereitschaft von Patienten zu weiterer konventioneller Behandlung das Zusammenstellen von Vergleichsgruppen so ausserordentlich schwer macht, wünscht man sich von den Autoren eine detaillierte Beschreibung des Zustandekommens beider Gruppen, auch über die Stärke der Präferenz für ein Verfahren und über die (homöopathische) Vorgeschichte bleibt der Leser leider im Unklaren.

$\mathrm{Zu}$ guter Letzt erfordern die Ergebnisse dieser Studie eine Konsolidierung anhand anderer Beschwerden in der Allgemeinpraxis.

Kontaktadresse: Dipl.-Psych. Corina Güthlin, Institut für Umweltmedizin und Krankenhaushygiene, Hugstetter Straße 55, D-79106 Freiburg, E-mail guethlin@sun11.ukl.uni-freiburg.de

Adresse für Sonderdrucke: David Riley MD, Integrative Medicine Institute, P.O. Box 4310, Santa Fe, NM 87502, USA, E-mail dsriley@integrativemed.org. 